\title{
CROSSED PRODUCTS BY SEMIGROUPS OF ENDOMORPHISMS AND THE TOEPLITZ ALGEBRAS OF ORDERED GROUPS
}

\author{
SRIWULAN ADJI, MARCELO LACA, MAY NILSEN, AND IAIN RAEBURN
}

(Communicated by Palle E. T. Jorgensen)

\begin{abstract}
Let $\Gamma^{+}$be the positive cone in a totally ordered abelian group $\Gamma$. We construct crossed products by actions of $\Gamma^{+}$as endomorphisms of $C^{*}$ algebras, and give criteria which ensure a given representation of the crossed product is faithful. We use this to prove that the $C^{*}$-algebras generated by two semigroups $V, W: \Gamma^{+} \rightarrow B(H)$ of nonunitary isometries are canonically isomorphic, thus giving a new, self-contained proof of a theorem of Murphy, which includes earlier results of Coburn and Douglas.
\end{abstract}

\section{INTRODUCTION}

Let $\Gamma$ be a totally ordered abelian group with positive cone $\Gamma^{+}$. Murphy showed in [6] that the $C^{*}$-algebras generated by two semigroups $V, W: \Gamma^{+} \rightarrow$ $B(H)$ of nonunitary isometries are canonically isomorphic [6, Theorem 2.9], generalising well-known theorems of Coburn [3] for the case $\Gamma=\mathbb{Z}$, and of Douglas [5] for subgroups of $\mathbb{R}$. His proof involved an analysis of the ideal structure of the ordered group $\Gamma$, and used results of Olesen and Pedersen $[9, \S 8.11]$ on the primitivity of certain crossed products. Here we obtain a characterization of faithful representations of crossed products by semigroups of endomorphisms, and use it to give a short, self-contained proof of Murphy's theorem.

In $\S 1$, we discuss our notion of crossed products by semigroups of endomorphisms of $C^{*}$-algebras, which is based on ideas of Stacey [11]. Our characterization of faithful representations in Theorem 1.2 extends that for crossed products by single endomorphisms in [1]; we prove a more general theorem than we need later, because we want to fill a gap in [1] - see the remarks preceding Lemma 1.3. In $\S 2$, we show that the universal $C^{*}$-algebra $C^{*}\left(\Gamma^{+}\right)$for isometric representations of $\Gamma^{+}$is such a crossed product (Proposition 2.2). To prove the main result, Theorem 2.4, we need to verify that the hypotheses of Theorem 1.2 hold when the isometric representation consists of nonunitary isometries, and we do this by adapting an argument from [4]. It follows immediately that the Toeplitz algebra of an ordered group is isomorphic to $C^{*}\left(\Gamma^{+}\right)$.

Received by the editors March 17, 1993.

1991 Mathematics Subject Classification. Primary 46L55; Secondary 06F15, 47B35.

Key words and phrases. $C^{*}$-algebra, endomorphism, ordered group, covariant representation, crossed product, semigroup of isometries, Toeplitz algebra.

This research was supported by the Australian Research Council. 


\section{CROSSED PROducts}

Throughout $\Gamma$ will be a totally ordered discrete abelian group with positive cone $\Gamma^{+}$. An isometric representation of $\Gamma^{+}$is a homomorphism of the semigroup $\Gamma^{+}$into the semigroup of isometries $\operatorname{Isom}(H)$ on a Hilbert space $H$. We now give our basic definitions.

Definition. A dynamical system is a triple $\left(A, \Gamma^{+}, \alpha\right)$ where $A$ is a unital $C^{*}$-algebra, and $\alpha$ is an action of $\Gamma^{+}$on $A$ by endomorphisms. A covariant representation of a dynamical system $\left(A, \Gamma^{+}, \alpha\right)$ is a pair $(\pi, V)$, where $\pi$ is a nondegenerate representation of $A$ on $H$, and $V$ is an isometric representation of $\Gamma^{+}$on $H$ such that $\pi\left(\alpha_{x}(a)\right)=V_{x} \pi(a) V_{x}^{*}$ for $a \in A$ and $x \in \Gamma^{+}$. A crossed product for a dynamical system $\left(A, \Gamma^{+}, \alpha\right)$ is a unital $C^{*}$-algebra $B$ together with a unital homomorphism $i_{A}: A \rightarrow B$ and a homomorphism $i_{\Gamma^{+}}$of $\Gamma^{+}$into the semigroup of isometries in $B$ satisfying

1. $i_{A}\left(\alpha_{x}(a)\right)=i_{\Gamma^{+}}(x) i_{A}(a) i_{\Gamma^{+}}(x)^{*}$ for $a \in A, x \in \Gamma^{+}$;

2. for every covariant representation $(\pi, V)$ of $\left(A, \Gamma^{+}, \alpha\right)$, there is a unital representation $\pi \times V$ of $B$ with $(\pi \times V) \circ i_{A}=\pi$ and $(\pi \times V) \circ i_{\Gamma^{+}}=V$; and

3. $B$ is generated by $\left\{i_{A}(a)\right\}$ and $\left\{i_{\Gamma^{+}}(x)\right\}$.

Remark 1.1. (i) In fact, $B$ is spanned by $\left\{i_{\Gamma^{+}}(x)^{*} i_{A}(a) i_{\Gamma^{+}}(y): a \in A, x, y \in\right.$ $\left.\Gamma^{+}\right\}$; cf. [1, Lemma 1.1].

(ii) As in [10, Proposition 3] and [11, Proposition 3.2], each system has, up to isomorphism, exactly one crossed product, denoted by $\left(A \times_{\alpha} \Gamma^{+}, i_{A}, i_{\Gamma^{+}}\right)$.

(iii) If there is a covariant representation $(\pi, V)$ with $\pi$ faithful, condition 2 implies that $i_{A}$ is injective. However, there might not be such a representation; see $[11,2.2]$.

Theorem 1.2. Suppose $(\pi, V)$ is a covariant representation of $\left(A, \Gamma^{+}, \alpha\right)$ such that

(i) $\pi$ is faithful, and

(ii) for all finite subsets $F$ of $\Gamma^{+}$and all choices of $a_{x, y} \in A$,

$$
\left\|\sum_{x \in F} V_{x}^{*} \pi\left(a_{x, x}\right) V_{x}\right\| \leq\left\|\sum_{x, y \in F} V_{x}^{*} \pi\left(a_{x, y}\right) V_{y}\right\| .
$$

Then $\pi \times V$ is a faithful representation of $A \times{ }_{\alpha} \Gamma^{+}$.

Proof. Our strategy is a familiar one. The uniqueness of the crossed product gives a continuous action $\beta$ of the compact group $\widehat{\Gamma}$ on $A \times{ }_{\alpha} \Gamma^{+}$, characterised by $\beta_{\gamma}\left(i_{A}(a)\right)=i_{A}(a)$ and $\beta_{\gamma}\left(i_{\Gamma^{+}}(x)\right)=\gamma(x) i_{\Gamma^{+}}(x)$ ( $\beta$ is the dual action). The formula $\theta(b)=\int_{\widehat{\Gamma}} \beta_{\gamma}(b) d \gamma$ defines a norm-decreasing projection $\theta$ of $A \times{ }_{\alpha} \Gamma^{+}$ onto the fixed-point algebra $\left(A \times_{\alpha} \Gamma^{+}\right)^{\beta}$, which is faithful in the sense that if $\theta\left(b^{*} b\right)=0$ for some $b \in A \times_{\alpha} \Gamma^{+}$, then $b=0$ (e.g., see the proof of [1, Lemma 2.2]). Since $\int \gamma(y-x) d \gamma=0$ for $x \neq y$, we have

$$
\theta\left(\sum_{x, y} i_{\Gamma^{+}}(x)^{*} i_{A}\left(a_{x, y}\right) i_{\Gamma^{+}}(y)\right)=\sum_{x} i_{\Gamma^{+}}(x)^{*} i_{A}\left(a_{x, x}\right) i_{\Gamma^{+}}(x),
$$

and the inequality in (ii) above extends to $\|\pi \times V(\theta(b))\| \leq\|\pi \times V(b)\|$ for all $b \in A \times_{\alpha} \Gamma^{+}$. 
The theorem will follow once we show that $\pi \times V$ is faithful on the fixedpoint algebra $\left(A \times \times_{\alpha} \Gamma^{+}\right)^{\beta}=\theta\left(A \times{ }_{\alpha} \Gamma^{+}\right)$. For if $\pi \times V\left(b^{*} b\right)=0$, then $\pi \times$ $V\left(\theta\left(b^{*} b\right)\right)=0$; because $\pi \times V$ is faithful on the range of $\theta$, this forces $\theta\left(b^{*} b\right)=0$, and hence also $b=0$.

So we have only to prove that $\pi \times V$ is faithful on $\left(A \times_{\alpha} \Gamma^{+}\right)^{\beta}$. From (1.1) and the continuity of $\theta$, we deduce

$$
\left(A \times_{\alpha} \Gamma^{+}\right)^{\beta}=\overline{\operatorname{span}}\left\{i_{\Gamma^{+}}(x)^{*} i_{A}(a) i_{\Gamma^{+}}(x): a \in A, x \in \Gamma^{+}\right\} .
$$

If we had the extra condition

$$
i_{\Gamma^{+}}(x)^{*} i_{A}(A) i_{\Gamma^{+}}(x) \subset i_{A}(A),
$$

it would immediately follow that $\left(A \times{ }_{\alpha} \Gamma^{+}\right)^{\beta}=i_{A}(A)$, and $\pi \times V$ would be faithful there because $\pi=(\pi \times V) \circ i_{A}$ is faithful on $A$ by assumption (i). Since (1.3) holds in the situation of $\S 2$, the weaker version of Theorem 1.2 we have just proved would suffice there.

One reason we want to prove Theorem 1.2 in the stated generality is to complete the proof of Proposition 2.1 of [1], which is the special case $\Gamma^{+}=\mathbb{N}$, and where the extra generality was crucial for the proposed improvement to [8]; it was not checked in [1] that $\pi \times V$ is faithful on the fixed-point algebra. Lemma 1.5 will complete the proof of Theorem 1.2 by verifying this. First, we need a basic lemma about ideals of direct limits which is implicit in [2, Lemma 3.1].

Lemma 1.3. Let $C$ be a $C^{*}$-algebra, and $\left\{B_{\alpha}\right\}$ a family of $C^{*}$-subalgebras such that $C=\overline{\bigcup_{\alpha} B_{\alpha}}$. If $J$ is a closed ideal in $C$, then $J=\overline{\bigcup_{\alpha}\left(J \cap B_{\alpha}\right)}$.

Proof. Let $J_{\alpha}=J \cup B_{\alpha}$. We want to show that $J=\overline{\bigcup_{\alpha} J_{\alpha}}$. Obviously $\overline{\bigcup_{\alpha} J_{\alpha}} \subset$ $J$, so suppose $x \notin \overline{\bigcup_{\alpha} J_{\alpha}}$. Then $\inf _{y \in \cup J_{\alpha}}\|x-y\|=\epsilon>0$. Since $C=\overline{\bigcup_{\alpha} B_{\alpha}}$, we can choose $\lambda$ and $x_{\lambda} \in B_{\lambda}$ such that $\left\|x_{\lambda}-x\right\|<\frac{\epsilon}{3}$. Thus, for any $y$ in $J_{\lambda}$,

$$
\left\|x_{\lambda}-y\right\| \geq\|x-y\|-\left\|x-x_{\lambda}\right\| \geq \epsilon-\left\|x-x_{\lambda}\right\| \geq \epsilon-\frac{\epsilon}{3}=\frac{2 \epsilon}{3} .
$$

If $\rho: C \rightarrow C / J$ is the quotient map, then $\left.\operatorname{ker} \rho\right|_{B_{\lambda}}=J \cap B_{\lambda}=J_{\lambda}$, and we have

$$
\left\|\rho\left(x_{\lambda}\right)\right\|=\inf _{y \in J_{\lambda}}\left\|x_{\lambda}-y\right\| \geq \frac{2 \epsilon}{3},
$$

because $\rho$ induces an isomorphism of the $C^{*}$-algebra $B_{\lambda} / J_{\lambda}$ onto $\rho\left(B_{\lambda}\right)$ which is necessarily isometric. Then

$$
\|\rho(x)\|=\left\|\rho\left(x_{\lambda}\right)+\rho\left(x-x_{\lambda}\right)\right\| \geq\left\|\rho\left(x_{\lambda}\right)\right\|-\left\|\rho\left(x-x_{\lambda}\right)\right\| \geq \frac{2 \epsilon}{3}-\frac{\epsilon}{3},
$$

since $\rho$ is norm-decreasing. Hence $\rho(x) \neq 0$, and $x \notin \operatorname{ker} \rho=J$.

Remark 1.4. This is the proof of [2, Lemma 3.1], slightly modified to avoid using the hypothesis that the $B_{\alpha}$ form an increasing sequence. The finitedimensionality of $B_{n}$ was not used in [2] except to ensure that $B_{n}$ is a $C^{*}$ algebra. 
Lemma 1.5. Let $(\pi, V)$ be as in Theorem 1.2. Then $\pi \times V$ is faithful on $\left(A \times{ }_{\alpha} \Gamma^{+}\right)^{\beta}$.

Proof. We claim that $\pi \times V$ is faithful on $\operatorname{span}\left\{i_{\Gamma^{+}}(x)^{*} i_{A}(a) i_{\Gamma^{+}}(x): a \in A, x \in\right.$ $\left.\Gamma^{+}\right\}$, which is dense in $\left(A \times_{\alpha} \Gamma^{+}\right)^{\beta}$ by (1.2). Suppose $b=\sum_{x} i_{\Gamma^{+}}(x)^{*} i_{A}\left(a_{x}\right) i_{\Gamma^{+}}(x)$ satisfies $\pi \times V(b)=0$, so that $\sum_{x} V_{x}^{*} \pi\left(a_{x}\right) V_{x}=0$. Choose $x_{0}$ greater than every $x$ for which $a_{x} \neq 0$. Then

$$
\begin{aligned}
0=V_{x_{0}}\left(\sum_{x} V_{x}^{*} \pi\left(a_{x}\right) V_{x}\right) V_{x_{0}}^{*} & =\sum_{x} V_{x_{0}-x} V_{x} V_{x}^{*} \pi\left(a_{x}\right) V_{x} V_{x}^{*} V_{x_{0}-x}^{*} \\
& =\sum_{x} V_{x_{0}-x} \pi\left(\alpha_{x}(1) a_{x} \alpha_{x}(1)\right) V_{x_{0}-x}^{*} \\
& =\sum_{x} \pi\left(\alpha_{x_{0}-x}\left(\alpha_{x}(1) a_{x} \alpha_{x}(1)\right)\right),
\end{aligned}
$$

so, since $\pi$ is faithful, $\sum_{x} \alpha_{x_{0}-x}\left(\alpha_{x}(1) a_{x} \alpha_{x}(1)\right)=0$ in $A$, and

$$
i_{A}\left(\sum_{x} \alpha_{x_{0}-x}\left(\alpha_{x}(1) a_{x} \alpha_{x}(1)\right)\right)=0 \text { in } A \times_{\alpha} \Gamma^{+} .
$$

Thus, reversing calculation (1.4), we obtain

$$
b=i_{\Gamma^{+}}\left(x_{0}\right)^{*}\left(i_{\Gamma^{+}}\left(x_{0}\right) b i_{\Gamma^{+}}\left(x_{0}\right)^{*}\right) i_{\Gamma^{+}}\left(x_{0}\right)=0,
$$

verifying the claim.

Next, let

$$
B_{z}=\operatorname{span}\left\{i_{\Gamma^{+}}(x)^{*} i_{A}(a) i_{\Gamma^{+}}(x): a \in A, 0 \leq x \leq z \in \Gamma^{+}\right\},
$$

so that $\bigcup_{z \in \Gamma^{+}} B_{z}$ is dense in $\left(A \times_{\alpha} \Gamma^{+}\right)^{\beta}$ by (1.2). We claim that each $B_{z}$ is a $C^{*}$-algebra. It is certainly a *-algebra, so it is enough to show $B_{z}$ is closed. Suppose $\left\{b_{n}\right\} \subset B_{z}$ and $b_{n} \rightarrow c$ in $A \times_{\alpha} \Gamma^{+}$. This implies that $i_{\Gamma^{+}}(z) b_{n} i_{\Gamma^{+}}(z)^{*} \rightarrow$ $i_{\Gamma^{+}}(z) c i_{\Gamma^{+}}(z)^{*}$. A calculation like (1.4), with $z$ in place of $x_{0}$, shows that each $i_{\Gamma^{+}}(z) b_{n} i_{\Gamma^{+}}(z)^{*}$ has the form $i_{A}\left(a_{n}\right)$ for some $a_{n}$ in $A$. Since $\left\{i_{A}\left(a_{n}\right)\right\}$ is Cauchy and the range of $i_{A}$ is closed, $i_{\Gamma^{+}}(z) b_{n} i_{\Gamma^{+}}(z)^{*}=i_{A}\left(a_{n}\right)$ converges to $i_{A}(a)$, for some $a$ in $A$. But then $b_{n}=i_{\Gamma^{+}}(z)^{*} i_{\Gamma^{+}}(z) b_{n} i_{\Gamma^{+}}(z)^{*} i_{\Gamma^{+}}(z)$ converges to the element $i_{\Gamma^{+}}(z)^{*} i_{A}(a) i_{\Gamma^{+}}(z)$ of $B_{z}$, and $B_{z}$ is closed. We can now apply Lemma 1.3 to deduce that $\operatorname{ker}(\pi \times V)=\overline{\bigcup_{z} \operatorname{ker}(\pi \times V) \cap B_{z}}$. Since we proved in the first paragraph that $\pi \times V$ is faithful on $\bigcup_{z} B_{z}$, we have

$$
\bigcup_{z} \operatorname{ker}(\pi \times V) \cap B_{z}=\operatorname{ker}(\pi \times V) \cap\left(\bigcup_{z} B_{z}\right)=\{0\} .
$$

This completes the proofs of Lemma 1.5 and Theorem 1.2.

\section{THE $C^{*}$-ALGEBRA GENERATED BY A SEMIGROUP OF ISOMETRIES}

We now consider a particular crossed product which is universal for isometric representations of $\Gamma^{+}$, in a sense to be made precise in Proposition 2.2. The algebra in the dynamical system is the closed subspace $B_{\Gamma^{+}}$of $\ell^{\infty}(\Gamma)$ spanned 
by $\left\{1_{x}: x \in \Gamma^{+}\right\}$, where

$$
1_{x}(y)= \begin{cases}1 & \text { if } y \geq x \\ 0 & \text { otherwise }\end{cases}
$$

since we have $1_{x} 1_{y}=1_{\max \{x, y\}}$, this space is actually a $C^{*}$-subalgebra of $\ell^{\infty}\left(\Gamma^{+}\right)$. For each $x \in \Gamma^{+}$, the automorphism $\tau_{x} \in$ Aut $\ell^{\infty}(\Gamma)$ defined by $\tau_{x}(f)(y)=f(y-x)$ satisfies $\tau_{x}\left(1_{y}\right)=1_{x+y}$, and hence $\tau$ restricts to an action $\alpha$ of $\Gamma^{+}$by endomorphisms of $B_{\Gamma^{+}}$. (The algebra $B_{\Gamma^{+}}$was introduced in [5], and is the restriction to $\Gamma^{+}$of the algebra $\mathscr{S}_{1}(\Gamma)$ used in [6].) Before proving that $B_{\Gamma^{+}} \times_{\alpha} \Gamma^{+}$has the required universal property, we need a lemma.

Lemma 2.1. Let $P_{i}$ be a finite family of projections such that $P_{1} \geq P_{2} \geq \cdots \geq P_{N}$. Then for any $\lambda_{i} \in \mathbb{C}$,

$$
\left\|\sum_{i=1}^{N} \lambda_{i} P_{i}\right\| \leq \max _{1 \leq n \leq N}\left|\sum_{i=1}^{n} \lambda_{i}\right| ;
$$

we have equality if $P_{i} \neq P_{i+1}$ for all $i$.

Proof. Since the projections $P_{n}-P_{n+1}$ are mutually orthogonal, the result follows from the identity

$$
\sum_{i=1}^{N} \lambda_{i} P_{i}=\sum_{n=1}^{N-1}\left(\sum_{i=1}^{n} \lambda_{i}\right)\left(P_{n}-P_{n+1}\right)+\left(\sum_{i=1}^{N} \lambda_{i}\right) P_{N} .
$$

Proposition 2.2. Let $\Gamma$ be a totally ordered abelian group and let $B_{\Gamma^{+}} \times_{\alpha} \Gamma^{+}$be the crossed product of the system defined above.

(i) If $\rho$ is a nondegenerate representation of $B_{\Gamma^{+}} \times_{\alpha} \Gamma^{+}$, then $\rho \circ i_{\Gamma^{+}}$is an isometric representation of $\Gamma^{+}$.

(ii) Whenever $V: \Gamma^{+} \rightarrow \operatorname{Isom}(H)$ is an isometric representation of $\Gamma^{+}$, there is a representation $\pi_{V}$ of $B_{\Gamma^{+}}$such that $\left(\pi_{V}, V\right)$ is a covariant representation of $\left(B_{\Gamma^{+}}, \Gamma^{+}, \alpha\right)$. If each $V_{x}$ is nonunitary, then $\pi_{V}$ is faithful.

(iii) $B_{\Gamma^{+}} \times_{\alpha} \Gamma^{+}$is generated by $\left\{i_{\Gamma^{+}}(x): x \in \Gamma^{+}\right\}$- indeed,

$$
B_{\Gamma^{+}} \times_{\alpha} \Gamma^{+}=\overline{\operatorname{span}}\left\{i_{\Gamma^{+}}(x) i_{\Gamma^{+}}(y)^{*}: x, y \in \Gamma^{+}\right\} \text {. }
$$

(iv) $i_{\Gamma_{\Gamma^{+}}}: B_{\Gamma^{+}} \rightarrow B_{\Gamma^{+}} \times_{\alpha} \Gamma^{+}$is injective.

Proof. The first part is clear because each $i_{\Gamma^{+}}(x)$ is an isometry. For (ii), we note that the representation $\pi_{V}$ must satisfy

$$
\pi_{V}\left(\sum_{x \in F} \lambda_{x} 1_{x}\right)=\left(\sum_{x \in F} \lambda_{x} \pi_{V}\left(\alpha_{x}(1)\right)\right)=\sum_{x \in F} \lambda_{x} V_{x} V_{x}^{*}
$$

We show that this formula gives a well-defined linear map $\pi_{V}$ on $\operatorname{span}\left\{1_{x}\right.$ : $\left.x \in \Gamma^{+}\right\}$, and simultaneously that $\pi_{V}$ extends to $B_{\Gamma^{+}}$, by showing that

$$
\left\|\sum \lambda_{x} V_{x} V_{x}^{*}\right\| \leq\left\|\sum \lambda_{x} 1_{x}\right\|
$$

Given a finite linear combination $\sum_{x \in F} \lambda_{x} 1_{x}$, we can index $F$ so that $x_{1}<$ $x_{2}<\cdots<x_{N}$ because $\Gamma$ is totally ordered. Then $\sum_{x} \lambda_{x} 1_{x}=\sum_{i=1}^{N} \lambda_{x_{i}} 1_{x_{i}}$ is a linear combination of projections such that $1_{x_{1}}>1_{x_{2}}>\cdots>1_{x_{N}}$, and 
Lemma 2.1 implies

$$
\left\|\sum_{x \in F} \lambda_{x} 1_{x}\right\|=\max _{1 \leq n \leq N}\left|\sum_{i=1}^{n} \lambda_{x_{i}}\right|
$$

For $y>x$,

$$
V_{x} V_{x}^{*}-V_{y} V_{y}^{*}=V_{x} V_{x}^{*}-V_{x} V_{y-x} V_{y-x}^{*} V_{x}^{*}=V_{x}\left(1-V_{y-x} V_{y-x}^{*}\right) V_{x}^{*},
$$

so $V_{x} V_{x}^{*} \geq V_{y} V_{y}^{*}$. Thus $\sum_{x \in F} \lambda_{x} V_{x} V_{x}^{*}$ is a linear combination of projections $V_{x_{i}} V_{x_{i}}^{*}$ such that $V_{x_{1}} V_{x_{1}}^{*} \geq V_{x_{2}} V_{x_{2}}^{*} \geq \cdots \geq V_{x_{N}} V_{x_{N}}^{*}$, and Lemma 2.1 gives

$$
\left\|\sum_{x \in F} \lambda_{x} V_{x} V_{x}^{*}\right\|=\left\|\sum_{i=1}^{n} \lambda_{x_{i}} V_{x_{i}} V_{x_{i}}^{*}\right\| \leq \max _{1 \leq n \leq N}\left|\sum_{i=1}^{n} \lambda_{x_{i}}\right|=\left\|\sum_{x \in F} \lambda_{x} 1_{x}\right\|,
$$

as claimed. Finally, $\pi_{V}$ is a ${ }^{*}$-homomorphism because $1_{x} 1_{y}=1_{\max \{x, y\}}$ and $V_{x} V_{x}^{*} V_{y} V_{y}^{*}=V_{\max \{x, y\}} V_{\max \{x, y\}}^{*}$. It follows easily from the formula $\alpha_{x}\left(1_{y}\right)=$ $1_{x+y}$ that $\left(\pi_{V}, V\right)$ is covariant on $\operatorname{span}\left\{1_{x}: x \in \Gamma^{+}\right\}$, and hence, by continuity, on $B_{\Gamma^{+}}$.

Suppose now that $V$ is a nonunitary representation. Then for $y>x, 1-$ $V_{y-x} V_{y-x}^{*}$ is a nonzero projection, (2.1) gives $V_{x} V_{x}^{*}>V_{y} V_{y}^{*}$, and Lemma 2.1 gives equality in (2.2). Thus in this case $\pi_{V}$ is actually isometric, and hence faithful on $B_{\Gamma^{+}}$. This proves (ii).

The elements $i_{\Gamma^{+}}(x)^{*} i_{B_{\Gamma^{+}}}\left(1_{y}\right) i_{\Gamma^{+}}(z)=i_{\Gamma^{+}}(x)^{*} i_{\Gamma^{+}}(y) i_{\Gamma^{+}}(y)^{*} i_{\Gamma^{+}}(z)$ span a dense subspace of $B_{\Gamma^{+}} \times_{\alpha} \Gamma^{+}$. We claim that, for any $x, y, z \in \Gamma^{+}$,

$$
i_{\Gamma^{+}}(x)^{*} i_{\Gamma^{+}}(y) i_{\Gamma^{+}}(y)^{*} i_{\Gamma^{+}}(z)=i_{\Gamma^{+}}(a) i_{\Gamma^{+}}(b)^{*},
$$

for some $a, b \in \Gamma^{+}$. There are six cases which need to be considered separately, and in each case we can write down formulas for $a$ and $b$. For example, if $z \leq y \leq x$, writing $i$ for $i_{\Gamma^{+}}$,

$$
\begin{aligned}
i(x)^{*} i(y) i(y)^{*} i(z) & =(i(y) i(x-y))^{*} i(y)(i(z) i(y-z))^{*} i(z) \\
& =i(x-y)^{*} i(y-z)^{*} \\
& =(i(y-z) i(x-y))^{*}=i(x-z)^{*}
\end{aligned}
$$

so $a=0$ and $b=x-z$ will do. The other orderings of $x, y$, and $z$ can be handled similarly, and (iii) follows. To prove (iv), it is enough by Remark 1.1(iii) to produce an isometric representation $V$ of $\Gamma^{+}$for which $\pi_{V}$ is faithful, and hence, by (ii), enough to produce one consisting of nonunitary isometries. The semigroup $T$ on $\ell^{2}\left(\Gamma^{+}\right)$defined by $T_{x}\left(\delta_{y}\right)=\delta_{y+x}$ will do.

Remark 2.3. Parts (i) and (ii) of Proposition 2.2 say that $B_{\Gamma^{+}} \times_{\alpha} \Gamma^{+}$has the universal property which characterises the $C^{*}$-algebra $C^{*}\left(\Gamma^{+}\right)$of the semigroup $\Gamma^{+}$, as studied in [7] (see [7, p. 324]).

Theorem 2.4. Let $\Gamma$ be a totally ordered abelian group and $V: \Gamma^{+} \rightarrow \operatorname{Isom}(H)$ an isometric representation of $\Gamma^{+}$. Then the representation $\pi_{V} \times V$ of Proposition 2.2(ii) is an isomorphism of $C^{*}\left(\Gamma^{+}\right)=B_{\Gamma^{+}} \times_{\alpha} \Gamma^{+}$onto $C^{*}\left(V_{x}: x \in \Gamma^{+}\right)$if and only if $V$ is nonunitary.

Proof. Since $\pi_{V} \times V\left(i_{\Gamma^{+}}(x)\right)=V_{x}$, and $\left\{i_{\Gamma^{+}}(x): x \in \Gamma^{+}\right\}$generates $B_{\Gamma^{+}} \times_{\alpha} \Gamma^{+}$, the range of $\pi_{V} \times V$ is precisely $C^{*}\left(V_{x}: x \in \Gamma^{+}\right)$. If $V_{z}$ is unitary for some 
nonzero $z$, then $\pi_{V} \times V\left(i_{B_{\Gamma^{+}}}\left(1-1_{z}\right)\right)=\pi_{V}\left(1-1_{z}\right)=1-V_{z} V_{z}^{*}=0$. Since $i_{B_{\Gamma^{+}}}$is injective and $1-1_{z} \neq 0$, this shows that $\pi_{V} \times V$ is not faithful.

Suppose now that $V$ is nonunitary. Then Proposition 2.2 says that $\pi_{V}$ is faithful, so by Theorem $1.2, \pi_{V} \times V$ will be faithful if

$$
\left\|\sum_{x \in H} V_{x}^{*} \pi_{V}\left(f_{x, x}\right) V_{x}\right\| \leq\left\|\sum_{x, y \in H} V_{x}^{*} \pi_{V}\left(f_{x, y}\right) V_{y}\right\|
$$

for all finite subsets $H$ of $\Gamma^{+}$and all choices of $f_{x, y} \in B_{\Gamma^{+}}$. Since $\operatorname{span}\left\{1_{y}: y \in\right.$ $\left.\Gamma^{+}\right\}$is dense in $B_{\Gamma^{+}}$, it suffices to show

$$
\left\|\sum \mu_{x, y, x} V_{x}^{*} V_{y} V_{y}^{*} V_{x}\right\| \leq\left\|\sum \mu_{x, y, z} V_{x}^{*} V_{y} V_{y}^{*} V_{z}\right\|
$$

for all $\mu_{x, y, z} \in \mathbb{C}$. As in (2.3), for each choice of $x, y, z \in \Gamma^{+}$, there are $a, b \in \Gamma^{+}$such that

$$
V_{x}^{*} V_{y} V_{y}^{*} V_{z}=V_{a} V_{b}^{*}
$$

an inspection of the cases shows that, because each $V_{x}$ is nonunitary, $a=$ $b$ precisely when $x=z$. Thus it is enough to show $\left\|\sum_{x \in F} \lambda_{x, x} V_{x} V_{x}^{*}\right\|$ $\leq\left\|\sum_{x, y \in F} \lambda_{x, y} V_{x} V_{y}^{*}\right\|$ for all finite subsets $F$ of $\Gamma^{+}$and $\lambda_{x, y} \in \mathbb{C}$. To do this, we borrow an idea from the proof of [4, Proposition 1.7]: given $\sum_{x, y \in F} \lambda_{x, y} V_{x} V_{y}^{*}$, we construct a projection $Q$ such that

$$
Q V_{x} V_{y}^{*} Q=0 \quad \text { for } x, y \in F \text { with } x \neq y,
$$

and

$$
\left\|Q\left(\sum_{x \in F} \lambda_{x, x} V_{x} V_{x}^{*}\right) Q\right\|=\left\|\sum_{x \in F} \lambda_{x, x} V_{x} V_{x}^{*}\right\|
$$

so that

$$
\begin{aligned}
\left\|\sum_{x, y} \lambda_{x, y} V_{x} V_{y}^{*}\right\| & \geq\left\|Q\left(\sum_{x, y} \lambda_{x, y} V_{x} V_{y}^{*}\right) Q\right\|=\left\|Q\left(\sum_{x} \lambda_{x, x} V_{x} V_{x}^{*}\right) Q\right\| \\
& =\left\|\sum_{x} \lambda_{x, x} V_{x} V_{x}^{*}\right\| .
\end{aligned}
$$

By Lemma 2.1 we can find $x_{M} \in F$ such that

$$
\left\|\sum_{x \in F} \lambda_{x, x} V_{x} V_{x}^{*}\right\|=\left|\sum_{x \leq x_{M}} \lambda_{x, x}\right| \text {. }
$$

Let $\delta=\min \{x-y: x, y \in F$ and $x>y\}$, and take $Q=V_{x_{M}}\left(1-V_{\delta} V_{\delta}^{*}\right) V_{x_{M}}^{*}$. We prove condition (2.5) first. For $x \in F, x>x_{M}$ implies $x \geq x_{M}+\delta$, and hence

$$
\begin{aligned}
Q V_{x} V_{x}^{*} & =V_{x_{M}} V_{x_{M}}^{*} V_{x} V_{x}^{*}-V_{x_{M}+\delta} V_{x_{M}+\delta}^{*} V_{x} V_{x}^{*} \\
& = \begin{cases}V_{x} V_{x}^{*}-V_{x} V_{x}^{*}=0 & \text { if } x>x_{M}, \\
V_{x_{M}} V_{x_{M}}^{*}-V_{x_{M}+\delta} V_{x_{M}+\delta}^{*}=Q & \text { if } x \leq x_{M} .\end{cases}
\end{aligned}
$$

By (2.6) it follows that

$$
\left\|Q\left(\sum_{x \in F} \lambda_{x, x} V_{x} V_{x}^{*}\right) Q\right\|=\left\|\sum_{x \leq x_{M}} \lambda_{x, x} Q\right\|=\left|\sum_{x \leq x_{M}} \lambda_{x, x}\right|=\left\|\sum_{x \in F} \lambda_{x, x} V_{x} V_{x}^{*}\right\|,
$$


as required. To prove condition (2.4), we note from (2.7) that $Q V_{y} V_{y}^{*}$ is selfadjoint for every $y \in F$, so $Q$ commutes with $V_{y} V_{y}^{*}$. Thus

$$
Q V_{x} V_{y}^{*} Q= \begin{cases}Q V_{x-y} V_{y} V_{y}^{*} Q=Q V_{x-y} Q V_{y} V_{y}^{*} & \text { if } x>y, \\ Q V_{y-x}^{*} V_{y} V_{y}^{*} Q=Q V_{y-x}^{*} Q V_{y} V_{y}^{*} & \text { if } x<y,\end{cases}
$$

and it is enough to show $Q V_{x-y} Q=0$ whenever $x>y$ and $x, y \in F$. But

$$
\begin{aligned}
Q V_{x-y} Q & =V_{x_{M}}\left(1-V_{\delta} V_{\delta}^{*}\right) V_{x_{M}}^{*} V_{x-y} V_{x_{M}}\left(1-V_{\delta} V_{\delta}^{*}\right) V_{x_{M}}^{*} \\
& =V_{x_{M}}\left(1-V_{\delta} V_{\delta}^{*}\right) V_{x-y}\left(1-V_{\delta} V_{\delta}^{*}\right) V_{x_{M}}^{*},
\end{aligned}
$$

which equals zero because $\left(1-V_{\delta} V_{\delta}^{*}\right) V_{x-y}=V_{x-y}-V_{\delta} V_{x-y-\delta}=0$. This completes the proof of Theorem 2.4 .

Corollary 2.5 [6, Theorem 2.9]. Let $\Gamma$ be a totally ordered abelian group, and $V, W$ two representations of $\Gamma^{+}$as nonunitary isometries on Hilbert space. Then the map $V_{x} \mapsto W_{x}$ extends to an isomorphism of $C^{*}\left(V_{x}: x \in \Gamma^{+}\right)$onto $C^{*}\left(W_{x}: x \in \Gamma^{+}\right)$.

Proof. Theorem 2.4 gives us isomorphisms $\pi_{V} \times V$ of $B_{\Gamma^{+}} \times_{\alpha} \Gamma^{+}$onto $C^{*}\left(V_{x}: x \in \Gamma^{+}\right)$such that $\pi_{V} \times V\left(i_{\Gamma^{+}}(x)\right)=V_{x}$, and $\pi_{W} \times W$ of $B_{\Gamma^{+}} \times_{\alpha} \Gamma^{+}$ onto $C^{*}\left(W_{x}: x \in \Gamma^{+}\right)$such that $\pi_{W} \times W\left(i_{\Gamma^{+}}(x)\right)=W_{x}$. Then $\left(\pi_{W} \times W\right)$ $\circ\left(\pi_{V} \times V\right)^{-1}$ is the isomorphism we want.

Definition. The family $\left\{\epsilon_{x}: x \in \Gamma\right\}$ of evaluation maps is an orthonormal basis for $L^{2}(\widehat{\Gamma})$, and the Hardy space $H^{2}\left(\Gamma^{+}\right)$is by definition the closed linear span of $\left\{\epsilon_{x}: x \in \Gamma^{+}\right\}$. Let $P$ denote the projection of $L^{2}$ onto $H^{2}$. For each $\phi \in C(\widehat{\Gamma})$, the Toeplitz operator $T_{\phi}$ with symbol $\phi$ is the operator on $H^{2}\left(\Gamma^{+}\right)$ defined by $T_{\phi}(f)=P(\phi f)$. The Toeplitz algebra $\mathscr{T}(\Gamma)$ of the totally ordered abelian group $\Gamma$ is the $C^{*}$-subalgebra of $B\left(H^{2}\left(\Gamma^{+}\right)\right)$generated by the Toeplitz operators $\left\{T_{\phi}: \phi \in C(\widehat{\Gamma})\right\}$.

Corollary 2.6 [6, Theorem 3.14]. Let $\Gamma$ be a totally ordered abelian group and $V$ a representation of $\Gamma^{+}$as nonunitary isometries on Hilbert space. Then there is a unique isomorphism $\phi$ of $\mathscr{T}(\Gamma)$ onto $C^{*}\left(V_{x}: x \in \Gamma^{+}\right)$such that $\phi\left(T_{\epsilon_{x}}\right)=V_{x}$ for all $x \in \Gamma^{+}$.

Proof. Since the map $\phi \mapsto T_{\phi}$ is *-linear, the Stone-Weierstrass theorem implies that $\mathscr{T}(\Gamma)$ is generated by the semigroup $\left\{T_{\epsilon_{x}}: x \in \Gamma^{+}\right\}$. Since each $T_{\epsilon_{x}}$ is a nonunitary isometry, the result follows from Corollary 2.5 .

Our next corollary says that every nonzero ideal in $C^{*}\left(\Gamma^{+}\right)=B_{\Gamma^{+}} \times_{\alpha} \Gamma^{+}$has nonzero intersection with the copy of $B_{\Gamma^{+}}$. We believe that this will be a useful tool in understanding the ideal stucture of $C^{*}\left(\Gamma^{+}\right)$.

Corollary 2.7. Let $\Gamma$ be a totally ordered abelian group. If $I$ is a nonzero closed ideal in $C^{*}\left(\Gamma^{+}\right)=B_{\Gamma^{+}} x_{\alpha} \Gamma^{+}$, then there exists a nonzero $x \in \Gamma^{+}$with

$$
1-i_{\Gamma^{+}}(x) i_{\Gamma^{+}}(x)^{*} \in I \text {. }
$$

Proof. The quotient $\left(B_{\Gamma^{+}} x_{\alpha} \Gamma^{+}\right) / I$ is generated by the semigroup of isometries $i_{\Gamma^{+}}(x)+I$ with $x \in \Gamma^{+}$. Since $I$ is nonzero, the quotient map determined by $i_{\Gamma^{+}}(x) \mapsto i_{\Gamma^{+}}(x)+I$ is not an isomorphism. Thus, by Theorem 2.4 , there exists a nonzero $x \in \Gamma^{+}$for which $i_{\Gamma^{+}}(x)+I$ is unitary, that is, for which $1-i_{\Gamma^{+}}(x) i_{\Gamma^{+}}(x)^{*} \in I$. 
To finish, we shall discuss the relationship between these corollaries and other interesting results on $C^{*}\left(\Gamma^{+}\right)$in [6]. First, it follows immediately from Corollary 2.7 that $C^{*}\left(\Gamma^{+}\right)$is prime: indeed, the intersection of any two nonzero ideals meets $i_{B_{\Gamma^{+}}}\left(B_{\Gamma^{+}}\right)$(cf. [6, Theorem 2.11]). As Murphy points out, one can also prove this by showing that the Toeplitz representation is faithful and irreducible: the irreducibility can be proved directly (the result for partially ordered groups in [6, Theorem 3.13] is harder), the faithfulness follows from Corollary 2.6, and that primitive implies prime is an elementary argument. Second, the simplicity of the commutator ideal $\mathscr{C}(\Gamma)$ of $C^{*}\left(\Gamma^{+}\right)$for $\Gamma \subset \mathbb{R}$ follows from Corollary 2.5 , exactly as in $[5$, p. 147] and even more easily from Corollary 2.7; the same applies to Murphy's generalisation [6, Theorem 2.11]. Murphy also proved, conversely to Douglas's theorem, that $\mathscr{C}(\Gamma)$ simple implies $\Gamma \subset \mathbb{R}$; again, his result concerns partially ordered groups, and is relatively straightforward if one is only interested in totally ordered groups (cf. [6, p. 324325]).

\section{REFERENCES}

1. S. Boyd, N. Keswani, and I. Raeburn, Faithful representations of crossed products by endomorphisms, Proc. Amer. Math. Soc. 118 (1993), 427-436.

2. O. Bratteli, Inductive limits of finite-dimensional $C^{*}$-algebras, Trans. Amer. Math. Soc. 171 (1972), 195-234.

3. L. A. Coburn, The $C^{*}$-algebra generated by an isometry I, Bull. Amer. Math. Soc. 73 (1967), 722-726.

4. J. Cuntz, Simple $C^{*}$-algebras generated by isometries, Comm. Math. Phys. 57 (1977), 173-185.

5. R. G. Douglas, On the $C^{*}$-algebra of a one-parameter semigroup of isometries, Acta Math. 128 (1972), 143-152.

6. G. J. Murphy, Ordered groups and Toeplitz algebras, J. Operator Theory 18 (1987), 303326.

7. $\_$, Ordered groups and crossed products of $C^{*}$-algebras, Pacific J. Math. 148 (1991), 319-349.

8. W. L. Paschke, The crossed product by an endomorphism, Proc. Amer. Math. Soc. 80 (1980), 113-118.

9. G. K. Pedersen, $C^{*}$-algebras and their automorphism groups, Academic Press, London and New York, 1979.

10. I. Raeburn, On crossed products and Takai duality, Proc. Edinburgh Math. Soc. (2) 31 (1988), 321-330.

11. P. J. Stacey, Crossed products of $C^{*}$-algebras by endomorphisms, J. Austral. Math. Soc. Ser. A 54 (1993), 204-212.

Department of Mathematics, University of Newcastle, New South Wales 2308, AusTRALIA

E-mail address, M. Laca: marcelo@frey.newcastle.edu.au

E-mail address, I. Raeburn: iain@frey.newcastle.edu.au 